\title{
A Simple Proof of the Unconditional Security of Relativistic Quantum Cryptography
}

\author{
S.N.Molotkov and S.S.Nazin \\ Institute of Solid State of Russian Academy of Sciences \\ Chernogolovka, Moscow District, 142432, Russia
}

\begin{abstract}
A simple proof of the unconditional security of a relativistic quantum cryptosystem based on orthogonal states is proposed. Restrictions imposed by special relativity allow to substantially simplify the proof compared with the non-relativistic cryptosystems involving non-orthogonal states. Important for the proposed protocol is the spatio-temporal structure of the quantum states which is actually ignored in the non-relativistic protocols employing only the structure of the state space of the information carriers. As a consequence, the simplification arises because of the inefficiency of collective measurements which constitute the major problem in the non-relativistic case.
\end{abstract}

PACS numbers: 89.70.+c, 03.65.-w

\section{Introduction.}

In contrast to the classical cryptography based on the laws of classical physics, quantum cryptography employs the fundamental laws of non-relativistic quantum mechanics $[1,2]$. Security of classical cryptography is based on the unproved computational complexity of some functions, for example the discrete logarithm, which are assumed to be computed using a physical device operating according to the laws of classical physics. It is also implicitly assumed that the exchange of information between the legitimate users is performed employing the classical objects. Since the laws of classical physics do not prohibit simultaneous measurements of any dynamical variables of a classical system without any disturbances, it is impossible to reliably detect the eavesdropping attempts during the exchange of information between the legitimate users within the framework of classical physics. Therefore, the security of quantum cryptosystems cannot be based on the detection of eavesdropping attempts during the key distribution and can only be associated with the exponential computational complexity of some functions.

The laws of classical physics constitute only an approximate description of physical reality. A more accurate description is provided by the non-relativistic quantum mechanics. The quantum mechanics allow in principle to construct a computational device (quantum computer) which for some problems is more powerful than the classical computers. The factorization problem which is faced by eavesdropper when attempting to extract (compute) the key in some open key classical cryptosystems is unsolvable (possesses exponential complexity) for a classical computer, but becomes solvable (possesses only polynomial complexity) for a quantum computer [3]. Therefore, the laws of non-relativistic quantum mechanics do not allow the unconditionally secure information exchange using the available classical algorithms (the unconditional security is understood as the security following from the fundamental laws of nature rather than that based on computational complexity).

Prohibiting the unconditionally secure (in the above sense) classical cryptography, the quantum mechanics opens new possibilities for quantum cryptography which is based on reliable detection of the eavesdropping attempts guaranteed by the laws of quantum mechanics if the information is carried by quantum systems.

The non-relativistic quantum cryptography is based on the following two consequences of the postulates of non-relativistic quantum mechanics: 
- The unknown quantum state cannot be copied (no cloning theorem) [4].

- No information about the states belonging to a non-orthogonal basis can be obtained without disturbing them [5].

For the orthogonal states this restriction does not hold. Moreover, there exist no restrictions on the instantaneous distinguishability of the orthogonal states without any disturbance. Therefore the usage of orthogonal states in the non-relativistic quantum cryptosystems has not even been discussed.

The non-relativistic quantum cryptosystems actually do not employ the spatio-temporal structure of the quantum states (since both the no-cloning theorem and the statement concerning the impossibility of acquiring any information about the states belonging to a non-orthogonal basis without disturbing them have a completely general nature). Used in the non-relativistic quantum protocols are only the properties of the Hilbert state space of the information carriers. The effects of state propagation between two distant users are not taken into account explicitly since they are not important. To be more precise, the attempts to take into account the spatio-temporal structure of the states do not seem promising from the cryptographic point of view because of the absence of any restrictions on the maximum speed of information transfer in the non-relativistic theory.

A rigorous proof of the unconditional security of non-relativistic quantum protocols when both the eavesdropper and legitimate users are only limited by the laws of quantum mechanics presents a very difficult problem. Several proofs characterized by different levels of complexity and employing different assumptions have been proposed until now. However, because of the complexity of the problem, no generally accepted view on the proof of the unconditional security has yet evolved $[6,7,8,9]$.

Just as the classical physics, the non-relativistic quantum mechanics provides only an approximate description of nature. A more accurate description taking into account the spatio-temporal structure of quantum states and the restrictions imposed by general relativity is provided by the relativistic quantum theory which, because of the absence of any sensible interpretation of relativistic quantum mechanics, arises from the very outset as the quantum field theory.

The quantum field theory does not close the non-relativistic quantum cryptography because the states of quantum field systems are also described by the rays in physical Hilbert space [10], just as in the nonrelativistic quantum mechanics. Since the non-relativistic quantum cryptographic protocols employ only the properties of the states in the Hilbert space, the non-relativistic protocols survive in the quantum field theory. For the cryptographic problems the quantum field theory can only contribute a substantially new aspect if the spatio-temporal structure of the states is explicitly considered in the protocols. To be more precise, one should take into account that the quantum states, although being described by the rays in the Hilbert space just as in the non-relativistic quantum mechanics, are generated by the field operators (to be more precise, operator-valued distributions) which contain information about the structure of the space-time. The field operators satisfy the commutation relations expressing the microcausality principle. The latter follows from the restrictions imposed by special relativity according to which there should be no causal relation between any two points of Minkowski space-time separated by a space-like interval. In addition the field theory allows to explicitly take into account and employ in the construction of cryptographic protocols the effects of state propagation in the Minkowski space-time.

The restrictions imposed by the filed theory and special relativity result in substantial differences in some problems of quantum information theory (see details in Refs. [11,12]) compared with the nonrelativistic case.

Presented below is a simple example of an unconditionally secure relativistic quantum cryptosystem in a noisy channel employing the quantum field states (photons) as the information carriers. Because of more restrictive laws of the relativistic quantum field theory, the proof of the unconditional security is 
substantially simplified compared with the non-relativistic case. In addition, the proposed scheme can be rather easily realized experimentally, in contrast to the unconditionally secure exchange protocols in the non-relativistic case where the proof of security substantially employs the collective measurements which are very difficult for experimental realization. The proposed scheme explicitly employs the causality effects and only the individual measurements.

The below arguments are induced by the paper [13] which, in our opinion, has not been assessed correctly [14]. Further improvements [15] have in fact reduced it to a non-relativistic cryptosystem based on non-orthogonal states actually discarding the key new idea.

The key idea is to use the internal degrees of freedom of a quantized field (the photon helicity) to code the transmitted information while the spatial degrees of freedom are used to detect the eavesdropping attempts. This is a substantially new aspect compared with the non-relativistic case which, combined with the special relativity, allows to reliably detect any attempts by eavesdropper to delay the transmitted states and to guarantee that the eavesdropper obtains no information about them. The fact that the transmitted states are the states of a quantum field is also important for the protocol.

The quantum field theory allows to use even orthogonal states in the cryptosystems. Since in the relativistic case the system states are also described by the rays in the Hilbert state space just as in the non-relativistic quantum mechanics, both the no-cloning theorem and the statement on impossibility of reliable distinguishability of non-orthogonal states without disturbing them remain valid because their proofs employ only the properties of the Hilbert state space.

Important for further analysis are the following two circumstances dictated by the quantum field theory (see details in Refs. $[16,17]$ ):

- Reliable distinguishability of a pair of orthogonal states of a free quantum field requires access to the entire domain of the Minkowski space-time where the state supports are located. Formally, any two orthogonal states of a free quantum field can only be reliably distinguished without disturbing them in an infinite time because of the inherent unlocalizability of quantum field states (no field state with a compact support in the position space can be constructed whose support in the four-dimensional momentum space is defined on the mass shell).

- The theory allows the existence of free quantum field states arbitrarily strongly localized in the space-time (with any localization degree and decaying at infinity according to a law arbitrarily close to the exponential one).

The fundamental unlocalizability of the states is a consequence of the local nature of quantum field theory.

The latter circumstance is important for cryptography since it allows to prepare arbitrarily strongly localized states which have any required arbitrarily small tails decaying arbitrarily close to the exponential law beyond the space-time domains controlled by the legitimate users. The probability of distinguishing two orthogonal states of a quantum field can vary due to the effects of field propagation from the controlled domain of the Minkowski space-time to the domain accessible for the measurement procedure from $1 / 2$ (complete indistinguishability) to 1 (reliable distinguishability). To be more precise, due to the preparation of strongly localized field states, the probabilities of obtaining the measurement results in a finite time can be made arbitrarily close to $1 / 2$ or 1 , the deviation being the smallest parameter in the considered problem. Because of the restriction on the maximum speed of both field propagation and motion of classical objects (including measuring apparatus), the access to the entire domain of the existence of the field consisting of two arbitrarily strongly localized but spatially separated "halves" inevitably requires a finite time and cannot be performed instantly. Therefore, as the information is being acquired, the distinguishability probability changes from $1 / 2$ to 1 in a finite time interval. Strong 
localization of the field "halves" separated by $\tau_{d}$ allows to construct the protocol in such a way that the distinguishability probability $P(\tau)=1 / 2$ at $0 \leq \tau<\tau_{d}$ and then jumps to $P(\tau)=1$ at $\tau=\tau_{d}$ (the jump is smeared by the state localization length $\left.\Delta \tau \ll \tau_{d}\right)$. The jump smearing is controlled by the localization of each "halves" and can be made arbitrarily small.

Since the relativistic quantum cryptography explicitly employs the spatio-temporal structure of the states, the protocol security cannot be proved without taking the geometry of a specific cryptosystem into account, in contrast to the non-relativistic case where only the structure of the state space is used.

\section{Measurements used in the protocol.}

Consider now the measurements used in the protocol. Since we should necessarily take into account the specific geometry of the cryptosystem, we shall consider a simple one-dimensional model containing all the important features dictated by the field theory. We shall consider the particles (field quanta) moving with the speed of light and the spectrum residing on the one-dimensional mass shell (front part of the one-dimensional light cone in the momentum representation, $k^{2}-k_{0}^{2}=0$ ).

Each of the users $\mathrm{A}$ and $\mathrm{B}$ controls the neighbourhoods of points $x_{A, B}$ (see the figure). The sizes of the controlled domains are dictated by the localization degree of the states and can be made arbitrarily small (should be of the order of the state localization length). In this geometry it is sufficient to consider the states propagating from $x_{A}$ to $x_{B}$ with positive momenta $k>0$. All the states are defined on one part of the light cone $\tau=x-t(c=1)$. The Hilbert state space of the information carriers is $\mathcal{H}_{k} \otimes \mathbf{C}^{2}$, where $\mathcal{H}_{k}$ is related to the spatial while $\mathbf{C}^{2}$ to internal (polarizational) degrees of freedom.

Consider a pair of orthogonal states

$$
\left|\psi_{0,1}\right\rangle=\frac{1}{\sqrt{2}} \int_{0}^{\infty} f(k)|k\rangle \otimes(|+\rangle \pm|-\rangle) d k
$$

where $| \pm\rangle \in \mathbf{C}^{2}$ are the orthogonal basis states, and

$$
\left\langle k \mid k^{\prime}\right\rangle=\delta\left(k-k^{\prime}\right), \quad k, k^{\prime}>0, \quad \int_{0}^{\infty}|f(k)|^{2} d k=1 .
$$

In the position representation the states on the branch of the one-dimensional light cone have the form

$$
\begin{gathered}
\left|\psi_{0,1}\right\rangle=\frac{1}{\sqrt{2}} \int_{-\infty}^{\infty} f(\tau)|\tau\rangle \otimes(|+\rangle \pm|-\rangle) d \tau, \quad \tau=x-t \\
|\tau\rangle=\int_{0}^{\infty} \mathrm{e}^{-i k \tau}|k\rangle d k, \quad f(\tau)=\int_{0}^{\infty} \mathrm{e}^{i k \tau} f(k) d k
\end{gathered}
$$

The basis $\{|\tau\rangle\}$ is not orthogonal, $\left\langle\tau \mid \tau^{\prime}\right\rangle=\delta_{+}\left(\tau-\tau^{\prime}\right) \neq \delta\left(\tau-\tau^{\prime}\right)$.

The measurement allowing to reliably distinguish the specified pair of orthogonal states is given by an appropriate identity resolution in $\mathcal{H}_{k} \otimes \mathbf{C}^{2}$ (and, similarly, by a resolution in $\mathcal{H}_{\tau} \otimes \mathbf{C}^{2}$ where $\mathcal{H}_{\tau}$ is the space isomorphic to $\mathcal{H}_{k}$ spanned by the basis $\left.\{|\tau\rangle\}\right)$.

The measurement is given by

$$
\begin{gathered}
\mathcal{M}_{0}+\mathcal{M}_{1}=I_{k} \otimes I_{\mathbf{C}^{2}}, \quad \mathcal{M}_{0,1}=I_{k} \otimes \mathcal{P}_{0,1}=I_{\tau} \otimes \mathcal{P}_{0,1}, \\
\mathcal{P}_{0}=|0\rangle\left\langle 0\left|, \quad \mathcal{P}_{1}=\right| 1\right\rangle\langle 1|, \quad| 0\rangle=\frac{1}{\sqrt{2}}(|+\rangle+|-\rangle), \quad|1\rangle=\frac{1}{\sqrt{2}}(|+\rangle-|-\rangle),
\end{gathered}
$$




$$
\begin{gathered}
I_{k}=\int_{0}^{\infty}|k\rangle\left\langle k\left|d k=I_{\tau}=\int_{-\infty}^{\infty}\right| \tau\right\rangle\langle\tau| d \tau=\int_{-\infty}^{\infty} \mathcal{M}(d \tau), \\
\mathcal{M}(d \tau)=\left(\int_{0}^{\infty} \mathrm{e}^{-i k \tau}|k\rangle d k\right)\left(\int_{0}^{\infty} \mathrm{e}^{i k^{\prime} \tau}\left\langle k^{\prime}\right| d k^{\prime}\right) d \tau .
\end{gathered}
$$

Note that the measurement (4) is non-local on the light cone.

Support of the state $f(\tau)$ on the light cone can be chosen arbitrarily strongly localized, in the extreme case $|f(\tau)|^{2} \rightarrow \delta(\tau)$. Strictly speaking, in the field theory the states defined on the mass shell cannot have a compact support in the Minkowski space-time. However, one can construct arbitrarily strongly localized states with the tails arbitrarily close to the exponential ones [17]. The latter means that it is possible to choose a time window $\Delta \tau$ such that the probability of detecting the state in this window can be made arbitrarily close to unit. We shall assume that the state (actually the packet shape $f(\tau)$ ) and the time interval $\Delta \tau$ are chosen such that the probability of detecting the state outside the time window $\Delta \tau$ due to the state tails extending beyond $\Delta \tau$ can be made exponentially arbitrarily close to zero. In the rest of the paper we shall assume this parameter to be the smallest one in the problem. To be more precise, the probability of detecting the input states $\left|\psi_{0,1}\right\rangle$ in the time window $\Delta \tau$ in the channel 0 (the channel corresponding to $\left.\mathcal{P}_{0}\right)$ and, respectively, in the channel $1\left(\mathcal{P}_{1}\right)$ (see Eq.(4)) is

$$
\operatorname{Pr}\left\{\Delta \tau,\left|\psi_{0,1}\right\rangle\right\}=\operatorname{Tr}\left\{\left(\left(\int_{-\Delta \tau}^{\Delta \tau} \mathcal{M}(d \tau)\right) \otimes \mathcal{P}_{0,1}\right)\left|\psi_{0,1}\right\rangle\left\langle\psi_{0,1}\right|\right\}=\int_{-\Delta \tau}^{\Delta \tau}|f(\tau)|^{2} d \tau=1-\delta,
$$

where $\delta$ is only different from zero due to the state tails

$$
\int_{|\tau|>\Delta \tau}|f(\tau)|^{2} d \tau=\delta \rightarrow 0
$$

In other words, there are two different parameters in the problem: $\Delta \tau$, the typical state localization (the interval is chosen so that the squared amplitude state $f(\tau)$ integrated over it is arbitrarily close to unit) and $\tau_{d}$, the separation between the two "halves" of the state chosen to make the overlap between the tails of the two "halves" arbitrarily small $\left(\Delta \tau \ll \tau_{d}\right)$. In the further analysis we shall assume for convenience (with the above reservations) the support to be a compact set since this assumption will not affect the final result.

It should be noted here that the measurement under consideration cannot be interpreted as the one having duration $\Delta \tau$. In each measurement act the obtained result (reading of a classical device, e.g. of a fast photodetector with a small response time (in the limit, formally infinitely small) operating in the waiting mode) arises at a random time moment in the interval $(\tau, \tau+d \tau)$ with the probability density

$$
\operatorname{Pr}\left\{d \tau,\left|\psi_{0,1}\right\rangle\right\}=\operatorname{Tr}\left\{\left((\mathcal{M}(d \tau)) \otimes \mathcal{P}_{0,1}\right)\left|\psi_{0,1}\right\rangle\left\langle\psi_{0,1}\right|\right\}=|f(\tau)|^{2} d \tau .
$$

This interpretation is very natural and is consistent with the classical limit when a classical signal with the temporal shape $f(\tau)$ is measured.

Let the states $\left|\psi_{0}\right\rangle$ (or $\left|\psi_{1}\right\rangle$ ) are prepared at time $\tau_{i}$ (to within $\Delta \tau$; this requires the control over the spatio-temporal domain of size $\Delta \tau)$

$$
\left|\psi_{0,1}\right\rangle=\frac{1}{\sqrt{2}} \int_{-\infty}^{\infty}\left(f\left(\tau-\tau_{i}\right)|\tau\rangle \otimes(|+\rangle \pm|-\rangle)\right) d \tau
$$

and then a unitary transformation is performed which does not depend on the prepared state:

$$
\left|\psi_{0,1}\left(\tau_{d}\right)\right\rangle=U\left|\psi_{0,1}\right\rangle=\frac{1}{\sqrt{2}} \int_{-\infty}^{\infty}\left(f\left(\tau-\tau_{i}-\tau_{d}\right)|\tau\rangle \otimes|+\rangle \pm f\left(\tau-\tau_{i}\right)|\tau\rangle \otimes|-\rangle\right) d \tau
$$


The accuracy of the moment of preparation is determined by the width of the state support.

Matrix elements of the unitary operator have the form

$$
\begin{gathered}
\left\langle+\left|\left\langle\tau^{\prime}|U| \tau\right\rangle\right|+\right\rangle=\delta_{+}\left(\tau-\tau^{\prime}-\tau_{d}\right)=\int_{0}^{\infty} \mathrm{e}^{i k\left(\tau-\tau^{\prime}-\tau_{d}\right)} d k, \\
\left\langle-\left|\left\langle\tau^{\prime}|U| \tau\right\rangle\right|-\right\rangle=\delta_{+}\left(\tau-\tau^{\prime}\right), \quad\left\langle \pm\left|\left\langle\tau^{\prime}|U| \tau\right\rangle\right| \mp\right\rangle=0 .
\end{gathered}
$$

This unitary transformation is non-local on the light cone $\tau=x-t$ (for $\tau_{d} \neq 0$ ) which means that its realization requires access to a domain of the size $\tau_{d}$ (to within $\Delta \tau$ ) on the light cone. Physically, this unitary transformation corresponds to a shift (delay) of the "half" of the state with the $|-\rangle$ polarization along the light cone. If this transformation is performed at fixed $x$ (locally in the ordinary position space), then it requires time $\Delta t=\tau_{d}$ (since $\tau=x-t$ ), or it requires the spatial domain of size $\Delta x=c \tau_{d}$ $(c=1)$ if the transformation is performed at a fixed moment of time but non-locally in space (in a spatial domain of size $\Delta x=c \tau_{d}$ ).

The spatio-temporal interval $\tau_{d}$ on the branch of the light cone does not depend on the choice of the reference frame since the light cone is Lorentz-invariant. Hence the eavesdropper cannot use the twin paradox [16].

The extended states $\left|\psi_{0}\left(\tau_{d}\right)\right\rangle$ and $\left|\psi_{1}\left(\tau_{d}\right)\right\rangle$ are orthogonal.However, the orthogonality is a non-local property in the sense that to find out whether or not the two states are orthogonal one has to have access to the spatio-temporal domain (interval) of length $\geq \tau_{d}$ to within $\Delta \tau \rightarrow 0$. In other words, the orthogonality is also a non-local property in the Hilbert space $\mathcal{H}_{\tau}$ in the sense that one has to have access to all states $|\tau\rangle$ (access to the spatio-temporal domain $\tau \geq \tau_{d}$ ), on which the space $\mathcal{H}_{\tau}$ is spanned.

All the non-relativistic quantum cryptographic protocols assume that the entire Hilbert state spaces are always accessible to both the legitimate users and eavesdropper. In the relativistic case the access can be changed by the effects of the state propagation from the domain controlled by the legitimate user to the domain accessible to the eavesdropper. "Extension" of the state and the limitation on the maximum speed of motion of classical objects and propagation of quantum states allows to construct a protocol in such a way that the state is never available to the eavesdropper as a whole. To be more precise, the attempts to get access to the entire state requires access to the whole interval where the state is present. However, because of the finite speed of light the attempts to get access to a finite spatio-temporal domain result in inevitable delay in the state detection by a legitimate user. This is actually the reason why the collective measurements which are efficient in the non-relativistic case (because of the availability of the entire state space to all the participants) and which present the major obstacle in the proof of the unconditional security, are insignificant here. One can only consider the individual measurements in each transmission since the presence of eavesdropper is detected by the delay of state detection by a legitimate user in each event.

If one has access to the interval $2 T$ centered about $\tau_{0}, T_{0}=\left(-T+\tau_{0}, \tau_{0}+T\right)$ and $2 T<\tau_{d}+\Delta \tau$, then no measurement over the states $\left|\psi_{0}\left(\tau_{d}\right)\right\rangle$ and $\left|\psi_{1}\left(\tau_{d}\right)\right\rangle$ can distinguish between them (the states seem to be identical). The latter is formally expressed by the restriction of the density matrix to the subspace $\mathcal{H}_{T_{0}}$ spanned by the states $|\tau\rangle$ whose support belongs to the interval $T_{0}$. For the density matrix we have

$$
\begin{aligned}
& \rho_{T}=\operatorname{Tr}_{\tau}\left\{\left(\left(\int_{T_{0}} \mathcal{M}(d \tau)\right) \otimes I_{\mathbf{C}^{2}}\right)\left|\psi_{0,1}\left(\tau_{d}\right)\right\rangle\left\langle\psi_{0,1}\left(\tau_{d}\right)\right|\right\}= \\
& \frac{1}{2} \int_{T_{0}}|f(\tau)|^{2} d \tau \otimes|+\rangle\left\langle+\left.\left|+\frac{1}{2} \int_{T_{0}}\right| f\left(\tau-\tau_{d}\right)\right|^{2} d \tau \otimes \mid-\right\rangle\langle-| .
\end{aligned}
$$

If the interval $T_{0}<\tau_{d}+\Delta \tau$ does not cover simultaneously the supports of the states with different polarizations (see figure) then only one of the functions $f(\tau)$, or $f\left(\tau-\tau_{d}\right)$ is different from zero. Thus, 
no measurement in the spatio-temporal domain (interval $T_{0}<\tau_{d}+\Delta \tau$ ) can distinguish the orthogonal states. The probability of distinguishing the states is $1 / 2$ (equal to the probability of simply guessing). Because of the limitation on the maximum speed the access to the interval $\tau_{d}$ cannot be obtained faster than the length of the interval itself.

\section{Description of the protocol.}

Legitimate users $\mathrm{A}$ and $\mathrm{B}$ control the neighbourhoods of points $x_{A}$ and $x_{B}, x_{A}<x_{B}$ (see the figure). Their clocks are assumed to be synchronized. The size of the controlled neighbourhoods should be $\Delta x_{A, B} \sim \Delta \tau$. The state support width $\Delta \tau \rightarrow 0$ is assumed to be known and is the smallest parameter in the problem. The communication channel length $\left(x_{B}-x_{A}=\tau_{c h}\right)$ is also assumed to be known (however, the absolutely exact knowledge of $\tau_{c h}$ is not required).

1. User A prepares with equal probabilities one of the states corresponding to 0 or 1 at a random time $\tau_{i}$ (to within $\left.\Delta \tau\right)$

$$
\left|\psi_{0,1}\right\rangle=\frac{1}{\sqrt{2}} \int_{-\infty}^{\infty} f\left(\tau-\tau_{i}-\tau_{A}\right)|\tau\rangle \otimes(|+\rangle \pm|-\rangle) d \tau, \quad \tau_{A}=x_{A} .
$$

Formally, the integration over $d \tau$ in Eq. (9) is performed over the entire branch of the light cone, although actually only the basis vectors $|\tau\rangle$ from the interval $\Delta \tau$ are involved in the state formtaion.

2. The "half" of the state (with the component $|+\rangle$ ) is sent into the communication channel while the second "half" with the component $|-\rangle$ is delayed which is described by the unitary transformation $U_{A}\left(\tau_{d}\right)$ (this interpretation of the unitary transformation is natural since $U_{A}\left(\tau_{d}\right)$ has matrix elements relating the states shifted along the light cone only for the polarization component $|+\rangle$ )

$$
\left|\psi_{0,1}\left(\tau_{d}\right)\right\rangle=U_{A}\left(\tau_{d}\right)\left|\psi_{0,1}\right\rangle=\frac{1}{\sqrt{2}} \int_{-\infty}^{\infty}\left(f\left(\tau-\tau_{i}-\tau_{A}-\tau_{d}\right)|+\rangle \pm f\left(\tau-\tau_{i}-\tau_{A}\right)|-\rangle\right) \otimes|\tau\rangle d \tau
$$

The transformation is non-local, its realization in the neighbourhood of point $x_{A}$ requires time $\tau_{d}$ and does not depend on whether the state 0 or 1 is considered.

3. State propagation from $\mathrm{A}$ to $\mathrm{B}$ is formally described by a unitary translation $U\left(\tau_{c h}\right)$ along the branch of the light cone by the interval $\tau_{c h}\left(\tau_{c h}=x_{B}-x_{A}\right)$

$$
\begin{gathered}
\left|\psi_{0,1}\left(\tau_{c h}\right)\right\rangle=U\left(\tau_{c h}\right)\left|\psi_{0,1}\left(\tau_{d}\right)\right\rangle= \\
\frac{1}{\sqrt{2}} \int_{-\infty}^{\infty}\left(f\left(\tau-\tau_{i}-\tau_{A}-\tau_{d}-\tau_{c h}\right)|+\rangle \pm f\left(\tau-\tau_{i}-\tau_{A}-\tau_{c h}\right)|-\rangle\right) \otimes|\tau\rangle d \tau .
\end{gathered}
$$

4. User B performs a unitary transformation $U_{B}\left(-\tau_{d}\right)$ which does not depend on the input state which merges the "halves" of the states back together (the shift of the "half" with the component $|+\rangle$ from and towards $|-\rangle$ can be preformed using the beam splitters, mirrors, and delay line)

$$
U_{B}\left(-\tau_{d}\right)\left|\psi_{0,1}\left(\tau_{c h}\right)\right\rangle=\frac{1}{\sqrt{2}} \int_{-\infty}^{\infty} f\left(\tau-\tau_{i}-\tau_{A}-\tau_{c h}\right)(|+\rangle \pm|-\rangle) \otimes|\tau\rangle d \tau .
$$

Matrix elements of the operator $U_{B}\left(-\tau_{d}\right)$ are similar to those of $U_{A}\left(\tau_{d}\right)$, but with $\tau_{d}$ changed to $-\tau_{d}$

$$
\begin{gathered}
\left\langle+\left|\left\langle\tau^{\prime}\left|U_{B}\left(-\tau_{d}\right)\right| \tau\right\rangle\right|+\right\rangle=\delta_{+}\left(\tau-\tau^{\prime}+\tau_{d}\right) \\
\left\langle-\left|\left\langle\tau^{\prime}\left|U_{B}\left(-\tau_{d}\right)\right| \tau\right\rangle\right|-\right\rangle=\delta_{+}\left(\tau-\tau^{\prime}\right), \quad\left\langle \pm\left|\left\langle\tau^{\prime}\left|U_{B}\left(-\tau_{d}\right)\right| \tau\right\rangle\right| \mp\right\rangle=0 .
\end{gathered}
$$


5. After the transformation $U_{B}\left(-\tau_{d}\right)$ the user B preforms the measurement realizing the identity resolution given by Eqs. $(3,4)$. The space of all possible measurement outcomes (results) is the set $\Theta=$ $\{i, \tau: i=0,1 ; \tau \in(-\infty, \infty)\}$ (index $i=0,1$ describes the outcomes in channels 0 and 1 ),

$$
\int_{-\infty}^{\infty} \mathcal{M}(d \tau) \otimes\left(\mathcal{P}_{0}+\mathcal{P}_{1}\right)=I_{\tau} \otimes I_{\mathbf{C}^{2}}
$$

This measurement describes the probabilities of obtaining a result in the interval $\Delta \tau$ in the channel 0 or 1 which is given by the expression

$$
\operatorname{Pr}\{(\Delta \tau)\}=\int_{\Delta \tau}\left|f\left(\tau-\tau_{i}-\tau_{A}-\tau_{c h}\right)\right|^{2} d \tau
$$

The result is different from zero if the interval $\Delta \tau$ covers the state support. Because of the orthogonality of the states the outcomes in the detection channels of user B coincide identically with the values sent by user $\mathrm{A}$ (if the noise is neglected).

The choice $\tau_{d}>\tau_{c h}$ means that only a part of the state is always available in the channel (only a part of the state space $\mathcal{H}_{\tau}$ is accessible), the access to only a part of the state space guaranteeing that the information about the states being zero (the probability of distinguishing the states is $1 / 2$ ). Because of the restriction on the maximum propagation speed the access to the second "half" of the state requires access to the interval $\tau>\tau_{d}>\tau_{c h}$ which inevitably results in a delay of the detection moment by the legitimate user B.

6. User B announces through the public channel the detection time $\tau_{B}$ of the state (which is known to within $\Delta \tau \rightarrow 0$ because of the narrow support of $f(\tau)$ ). If the detection did not occur, the transmission is discarded. After user B announced the detection time, user A announces the sending time $\tau_{i}$. If the detection time obtained by user $\mathrm{B} \tau_{B}=\tau_{i}+\tau_{d}+\tau_{c h}$ (to within $\Delta \tau$ ), the attempt is accepted. If the detection time is found to by delayed (by more than $\geq \tau_{d}$ ), the attempt is discarded.

It is fundamentally important, that the absence of delay in the detection moment measured by user $B$ already guarantees that the eavesdropper has zero information about the states sent by user $A$ (the probability of distinguishing the states is 1/2). Deviation of this probability from $1 / 2$ is due to the exponential tails of the states which can be made arbitrarily small by separating the "halves" (increasing $\left.\tau_{d}\right)$. If the state $f(\tau)$ has a compact support, the absence of a delay means that the information obtained by eavesdropper is strictly zero.

7. In the accepted transmissions the eavesdropper has zero information. However, because of the noise in the channel (decoherence processes) the sequences of 0 and 1 possessed by legitimate are not yet identical. The differences can be caused both by the eavesdropper and by the natural noise. For example, the eavesdropper can detect the first "half" of the state which is being sent by user A. This detection requires time $\Delta \tau \rightarrow 0$ (hence the eavesdropper determines time $\tau_{i}$ ). Then immediately after the detection the eavesdropper arbitrarily prepares his state $f(\tau)$ which will not cause any delay for user $\mathrm{B}$ but result in the inconsistency with the state sent by user A that will be interpreted as a noise in the channel. It follows from Eq.(8) that the probability of determination of the state preparation time is $1 / 2$ (because of the accessibility of only a "half" of the state), the state detection itself giving to the eavesdropper zero information. The probability of guessing the state is $1 / 2$. The total probability of correct determination of the state in the channel is $1 / 2 \cdot 1 / 2=1 / 4$. It should be noted that the probability of correctly guessing the state by the eavesdropper in each transmission when he undertakes no attempt to obtain any information from the channel is $1 / 2$. At first glance this seems strange since the probability of correct determination of the state by the eavesdropper is lower by a factor of 2 when he has access to the channel than when he simply guesses the state without access to the communication 
channel. However, actually there is nothing strange in this fact since the simple guessing involves only two outcomes (0 or 1$)$. On the other hand, when detecting the state in the channel one should also determine the state preparation moment $\tau_{i}$ (so that this event is not discarded by the legitimate users) and the detection probability for a "half" of the state is $1 / 2$. Therefore, if the eavesdropper attempts to access the channel the probability of erroneous interpretation of the intercepted state (which is equal $3 / 4$ ) includes also the probability of erroneous determination of the very fact of sending any state at all in the considered time interval (which is equal 1/2): since the detection itself yields zero information about the state, one has to guess it and the probability of guessing is $1 / 2$.

Hence, if the legitimate users keep only the transmissions in which no delay in photon detection was observed (to be more prices, the delay did not exceed $\tau_{d}+\tau_{c h}$ ) they can be sure that the probability of correct identification of the state by the eavesdropper intruding into the communication channel does not exceed 1/4. The latter is twice as low as the probability of correctly guessing the state without intruding into the channel.

We have here a curious situation which does not take place in the non-relativistic case. If we remember that the aim of the eavesdropper is to obtain maximum information about the key simultaneously minimizing the probability of being detected by legitimate users, then in this sense his optimal strategy consists in simply guessing the state sent in each attempt (the access to the communication channel is not required). It is only sufficient to have access to the classical communication channel (in order to know the total number of accepted transmissions) which in all quantum cryptographic problems is always assumed to be public. In that case the probability of detecting the eavesdropper is of course zero since he does not disturb the channel. Attempts to intrude into the communication channel are only sensible if the probability of the state identification (restricted to the transmissions accepted by the legitimate users) exceeds the probability of simple guessing which is $1 / 2$.

Hence the maximum probability of correct identification by the eavesdropper of each transmission accepted by the legitimate users is $1 / 2$.

8. Now the only problem to be solved is to ensure that the legitimate users have identical keys. Consider first a noiseless channel. After the sending session is completed and $2 N$ transmissions are accepted, the users $\mathrm{A}$ and $\mathrm{B}$ perform $m<2 N$ rounds of random hashing (parity check with a random bit string, see details in Ref. [18]). The length of the original sequence of $2 N$ bits is reduced by 2 after each round. If the parity check succeeds in $m$ rounds, the probability of having at least one difference in the left strings of $2 N-2 m$ bits possessed by users A and B does not exceed $2^{-m}$ which means that users $\mathrm{A}$ and $\mathrm{B}$ have identical keys with the probability exponentially close to unit. The above analysis implies also that the probability for the eavesdropper to have complete information about the key without being detected does not exceed $2^{-2(N-m)}$.

Suppose now that the channel is noisy leading to the errors in the detection results obtained by user $\mathrm{B}$, for example, due to the polarization rotation and let the probability this process is $p<1$ (probability of user A sending 0 and user B detecting 1 and vice versa). As in the previous case, the legitimate users accept only the transmissions where no delay was observed. After completing a series in which $4 N$ transmissions are accepted, users $\mathrm{A}$ and $\mathrm{B}$ disclose $2 N$ transmissions and estimate the noise level in the channel (probability $p$ ). Knowledge of the error in sending one bit allows in principle (for a sufficiently long sequence) to choose a suitable classical block code [19] which actually permits to reduce the error in code words to an arbitrarily small value.

For example, user A announces to B through a public channel only the numbers of transmissions where he sent 1 , combining them into the groups of $2 k$ transmissions, and similarly for 0 producing the blocks of size $2 k$ (coded 1 and 0 ). Then user B applies the majority voting to fix the errors in each group (this coding allows to fix $k-1$ errors). The blocks where $k$ errors were detected are rejected 
(user B announces the numbers of these blocks through the public channel). In the groups left after this procedure the error probability does not exceed $p^{k} \ll p$. Now the users have the sequences of the groups (new $\tilde{1}$ and $\tilde{0}$ ) of length $2 \tilde{N}$. Then the hashing procedure consisting of $m$ rounds similar to that described above is performed resulting in a sequence of length $2(\tilde{N}-m)$. The probability of users A and B having identical sequences of length $2(\tilde{N}-m)$ under the condition that $m$ rounds of random hashing gave successful parity tests is not less than $1-2^{-(\tilde{N}-m)}$. The block coding is only required to increase the surviving probability of the accepted sequence in the hashing procedure. The hashing procedure can also be applied to the original (rather than block) sequence. However, in that case the probability of missing the parity error during hashing will be small because of the noise. However, if the sequence passes the test, it is secure and identical for both users with the above probabilities. The probability of eavesdropper obtaining complete information about the key and remaining undetected is well below $2^{-2(\tilde{N}-m)}$ (because it is sufficient to guess one bit from each code group). This simple coding scheme obviously is not the optimal one, but it allows to formulate the cryptographic protocol in the simplest and most transparent way.

The authors are grateful to Prof. V.L.Golo for the interest to this work and discussion of the obtained results.

This work was supported by the Russian Foundation for Basic Research (project No 99-02-18127) as well as the project "Physical foundations of quantum computer" and Russian Federal Program "Advanced technologies and micro- and nanoelectronic devices" (project No 02.04.5.2.40.T.50).

\section{References}

[1] S.Wiesner, Conjugate coding, Sigact News. 15, 78 (1983).

[2] C.H.Bennett, G.Brassard, Quantum cryptography: Public key distribution and coin tossing, Proceedings of IEEE International Conference on Computers, Systems and Signal Processing, Bangalore, India, December 1984, p.175.

[3] P.W.Shor, Proceedings 35th Annual Symposium on Foundations of Computer Science, Santa Fe, NM, USA, ed by S.Goldwasser, (IEEE Comput. Soc. Press, Los Alamitos) 124 (1994).

[4] W.K.Wootters, W.H.Zurek, Nature, 299, 802 (1982).

[5] C.H.Bennett, Phys. Rev. Lett. 68, 3121 (1992); C.H.Bennett, G.Brassard, N.D.Mermin, Phys. Rev. Lett. 68, 557 (1992).

[6] D.Mayers, quant-ph/9802025.

[7] H.-K.Lo, H.F.Chau, quant-ph/9803006.

[8] E.Biham, M.Boyer, P.O.Boykin, T.Mor, V.Roychowdhury, quant-ph/9912053.

[9] P.W.Shor, J.Preskill, quant-ph/0003004.

[10] N.N.Bogolubov, A.A.Logunov, A.I.Oksak, I.T.Todorov, General principles of the quantum field theory, Moscow, "Nauka", 1987 (in Russian).

[11] A.Kent, quant-ph/9810067, quant-ph/9810068, Phys. Rev. Lett., 83, 1447 (1999). 
[12] S.N.Molotkov, S.S.Nazin, quant-ph/9911055, quant-ph/9910034; R.Laiho, S.N.Molotkov, S.S.Nazin, quant-ph/0005067, quant-ph/0005068.

[13] L.Goldenberg, L.Vaidman, Phys. Rev. Lett. 75, 1239 (1995); quant-ph/9506030.

[14] A.Peres, quant-ph/9509003.

[15] M.Koashi, N.Imoto, Phys. Rev. Lett. 79, 2383 (1997).

[16] R.Laiho, S.N.Molotkov, S.S.Nazin, quant-ph/00006010.

[17] I.Bialynicki-Birula, Phys. Rev. Lett., 80, 5247 (1998).

[18] C.H.Bennett, D.P.DiVincenzo, J.A.Smolin, W.K.Wootters, quant-ph/9604024.

[19] E.J.Mac Williams, N.J.A.Sloane, The Theory of Error-Correcting Codes, North-Holland Publ. Company, (Amsterdam, New York, Oxord), 1977. 




